\title{
OBESIDADE: COMPREENDENDO ESSE DESEQUILÍBRIO ORGÂNICO EM CÃES E GATOS
}

\author{
ANDRADE JÚNIOR, Antônio Gonçalves ${ }^{1}$; \\ CAPELLA, Sabrina Oliveira ${ }^{2}$; \\ PIÑEIRO, Martha Bravo $\mathrm{Cruz}^{3}$; \\ NOBRE, Márcia Oliveira ${ }^{4}$.
}

${ }^{1}$ Acadêmico da Faculdade de Veterinária da Universidade Federal de Pelotas (UFPEL); ${ }^{2}$ Médica Veterinária, Doutora, Universidade Federal de Pelotas; ${ }^{3}$ Pós-Graduanda do Programa de Pós-Graduação em Veterinária/UFPEL; ${ }^{4}$ Professora, Doutora, Departamento de Clínicas Veterinárias, Faculdade de Veterinária/UFPEL.

\section{RESUMO}

A obesidade é uma condição patológica caracterizada pelo acúmulo excessivo de gordura, sendo uma das principais patologias observadas em cães e gatos. É uma doença de prevalência crescente e influenciada por diversos fatores genéticos e ambientais, tais como: raça, idade, sexo, status gonadal, endocrinopatias, dieta e atividade física. É uma condição patológica que predispõe o animal a diversas complicações na saúde, como redução da longevidade, problemas em articulações, neoplasias e diabetes mellitus. Existem diversos métodos que auxiliam no diagnóstico dessa patologia e que são importantes para o tratamento. Diante disso, esta revisão tem por objetivo expor e discutir as possíveis etiologias e complicações, os métodos utilizados para o diagnóstico da obesidade e tratamento.

Palavras-chave: Sobrepeso. Distúrbio nutricional. Canídeos. Felídeos. 


\section{INTRODUÇÃO}

A obesidade é doença caracterizada pelo acúmulo excessivo de tecido adiposo no organismo do animal (DEBASTIANI, 2018), cabendo frisar que o tecido adiposo é um órgão endócrino ativo, que secreta uma gama de substâncias que desempenham um papel importante em diversos processos fisiológicos; todavia, o paciente obeso apresenta um estado constante de inflamação corporal, acarretando em uma série de desordens sistêmicas, consequentemente diminuindo a expectativa de vida (CARVALHO, 2014). A obesidade pode ser classificada como: hipertrófica, quando há uma hiperestimulação do tecido adiposo pelo excesso de gordura no organismo, geralmente observada em indivíduos adultos; e obesidade hiperplásica, ocasionada pela superalimentação energética observada em jovens devido ao crescimento (MENDES et al., 2013).

É necessário ressaltar que a obesidade é uma doença universal e de prevalência crescente, sendo um grave problema em saúde pública (SILVA et al., 2017). Segundo dados da Organização Mundial da Saúde, 39\% das mulheres e 39\% dos homens com 18 anos ou mais estavam com excesso de peso em 2016 (WHO, 2016). Um estudo realizado por Malta et al. (2014), em que foi avaliada a prevalência da população com excesso de peso nas capitais brasileiras, demonstrou que o excesso de peso na população adulta brasileira aumentou de $43,2 \%$ para $51,0 \%$ e a prevalência da obesidade aumentou de $11,6 \%$ para $17,4 \%$. Mas a obesidade não é, nos dias de hoje, apenas um assunto de saúde humana. A obesidade atravessou a fronteira das espécies e, tal como na população humana, a incidência da obesidade nos animais de companhia, cães e gatos, está aumentando (GERMAN, 2006; SILVA et al., 2017). De acordo com Nelson e Elliott (2006) tutores obesos constituem um fator adicional de risco para a obesidade nos animais de estimação, devido ao estilo de vida e pelo fato de não reconhecerem a obesidade como um problema em seus animais. Vários estudos no mundo relatam e documentam os índices crescentes da obesidade em cães e gatos (LUND et al., 2006; MAO et al., 2013; ROBERTSON, 2002), assim estima-se que 25 a $40 \%$ deles estejam obesos (CARVALHO, 2014).

A obesidade não se trata apenas da adiposidade, ou seja, acúmulo excessivo de tecido adiposo, mas está associada a importantes alterações metabólicas e hormonais no 
organismo (ZORAN, 2010). Essa doença predispõe os animais a distúrbios cardiorrespiratórios, articulares, diabetes mellitus, doenças do trato urinário, além de tumores e alterações na pele, visão e sistema imunológico (CARVALHO, 2014). A obesidade é uma doença multifatorial e pode ser influenciada por uma série de fatores de risco, sejam genéticos ou ambientais, fatores como a idade, sexo, estado reprodutivo, dieta, atividade fisica, entre outros, que desempenham um importante papel no desenvolvimento e manutenção da doença (DEBASTIANI, 2018).

Diante disso esta revisão tem por objetivo expor e discutir as possíveis etiologias e complicações, os métodos utilizados para o diagnóstico da obesidade e o tratamento.

\section{OBESIDADE EM CÃES E GATOS}

\section{Etiologia}

A obesidade pode ser de causa endógena, quando há uma endocrinopatia como o hipotireoidismo, exógena, devido à superalimentação ou o tipo de dieta, e mista, quando há um manejo alimentar errôneo associado a distúrbios endócrinos (FEITOSA, 2014). A causa exógena representa $95 \%$ dos casos atendidos, em que uma alta ingestão calórica diária associada a uma redução do gasto energético provoca um balanço energético positivo, favorecendo esse acúmulo de energia na forma de tecido adiposo, ressaltando que o uso de medicamentos como glicocorticoides ou fenobarbital também favorece o ganho de peso (CARVALHO, 2014).

A ingestão alimentar desempenha um papel importante para o desenvolvimento da obesidade, entretanto, outros fatores podem estar envolvidos, tais como a predisposição ao sobrepeso em determinadas raças de cães, como Labrador, Boxer, Basset Hound, Cocker Spaniel, Dachshund, Beagle, Golden Retriever e Rottweilers (BORGES, 2013; ZORAN, 2010). Da mesma forma, em felinos, foi observado que gatos da raça Manx foram os mais predispostos à obesidade (LUND et al., 2005). A influência de certas raças no metabolismo e na obesidade de cães e gatos é um pouco incerta (ZORAN, 2010), possivelmente essa influência se deva à diferença da composição corporal entre as raças, ou seja, na proporção entre massa magra e massa gorda e a fatores genéticos (JEUSETTE et al., 2010). Além disso, existem diferenças genéticas entre animais, o que determina necessidades energéticas 
variáveis para manter o peso ideal e isso demonstra que a constituição corpórea de determinadas raças desempenha um papel importante no desenvolvimento da obesidade (NELSON; ELLIOTT, 2006).

Além da raça, outro fator que predispõe os animais à obesidade é a idade, pois animais jovens apresentam um gasto energético maior devido a um metabolismo acelerado, associado aos processos anabólicos relativos ao crescimento (JERICÓ; SCHEFFER, 2002). Já os animais de meia idade a idosos são os mais propensos à obesidade, devido à diminuição do gasto energético. Como foi colocado, o metabolismo basal, que está relacionado com a composição corporal, desempenha um significativo papel no gasto energético e, com o avançar da idade, há uma diminuição do metabolismo basal, das necessidades energéticas diárias e, também, da atividade voluntária (SILVA et al., 2017).

O estado sexual é outro fator importante para obesidade, as fêmeas possuem uma taxa metabólica basal menor que os machos, por isso se tornam mais suscetíveis à obesidade (DEBASTIANI, 2018). Animais castrados tendem a ser mais obesos devido à desaceleração do metabolismo basal, gerando uma redução do gasto energético (LUND et al., 2005). Foi realizado um estudo epidemiológico, no qual foi demonstrado que em cães castrados a ocorrência de obesidade em fêmeas (67,3\%) foi maior que em machos (52,7\%) (MAO et al., 2013). Dessa forma, as fêmeas têm maior predisposição à obesidade, e quando são castradas, o risco aumenta, visto que o estrógeno exerce um efeito inibitório no apetite, logo, a ausência do efeito de hormônios gonadotróficos pode levar ao maior consumo de alimentos (SILVA et al., 2017).

Os animais que são sedentários são mais propensos à obesidade (MAO et al., 2013), pois a falta de atividade física gera um desequilíbrio no balanço energético, conduzindo a um balanço energético positivo em consequência do gasto energético reduzido (DEBASTIANI, 2018). Entretanto, a qualidade do exercício tem bastante influência na condição corporal do animal, pois foi demonstrado em um estudo que $50,4 \%$ dos animais que realizavam apenas caminhadas eram obesos, mas quando foi associada outra atividade física, a frequência reduziu para 40,1\% (BLAND et al., 2010). Com efeito, exercícios físicos com alta intensidade aumentam a taxa metabólica durante e depois do exercício, favorecendo o gasto energético 
(ROBERTSON, 2002). Entretanto, a mudança atual no estilo de vida dos cães e gatos, vivendo em espaços menores, sem atividades peculiares à espécie quando de vida livre, como por exemplo, a caça. Assim como a antropomorfização dos animais, associada ao estilo de vida prático e acelerado dos tutores, tem forte influência na condição corporal dos animais, pois contribuem para que esses animais não realizem atividade física (BORGES, 2013; SILVA et al., 2017).

A nutrição é um dos principais fatores relacionados com a manutenção da saúde e a condição corporal de cães e gatos (APTEKMANN et al., 2014). Nesse sentido, o tipo de dieta e outros fatores dietéticos têm forte influência no desenvolvimento e progressão do ganho de peso, como a alta densidade energética das dietas, o fornecimento de petiscos, sobras de alimentos, quantidade de alimento e o número de refeições ofertadas (BRUNETTO et al., 2011). É importante destacar que o expansivo mercado pet, com diversas opções de petiscos e/ou a falta de conhecimento e orientação do tutor sobre nutrição, favorecem o ganho de peso, já que alguns tutores têm o hábito de oferecer guloseimas e petiscos aos seus animais (CARVALHO, 2014; SILVA et al., 2017). Esses itens contêm alto teor de carboidratos e gordura, sendo que o nutriente que mais eleva o teor energético e a palatabilidade desses alimentos é a gordura, resultando em consumo energético diário além das necessidades do animal, induzindo o excesso de ganho de peso (BRUNETTO et al., 2011; DEBASTIANI, 2018). Como demonstrou o estudo de Mendes et al. (2013), no qual relata que a obesidade felina está mais relacionada a dietas ricas em gordura e com altos índices de energia metabolizável.

Estudos mostram que a frequência alimentar não é um fator de risco, devido à grande variabilidade dos resultados, mas uma frequência alimentar de 2 a 3 vezes por dia favorece o gasto energético (DEBASTIANI, 2018; PÖPPL, 2018). A quantidade de alimento oferecida reflete no ganho de peso, de acordo com Aptekmann et al. (2014) muitos tutores ainda estabelecem a quantidade de alimento a ser oferecida a seus cães de forma subjetiva. Logo, a ingestão alimentar diária excessiva, associada ao sedentarismo, produz um desequilíbrio entre o consumo e o gasto energético, o que conduz a um balanço energético positivo que aumenta o risco de acúmulo de gordura corporal (DEBASTIANI, 2018). 
Distúrbios endócrinos são fatores de risco importantes para a obesidade, como o hipotireoidismo, que produz um descenso do metabolismo basal, e o hiperadrenocorticismo, devido ao excesso de corticosteroides sintetizados; esses distúrbios levam a um aumento significativo na ingestão de alimentos e do peso corporal, predispondo o paciente à obesidade (SILVA et al., 2017). Ainda assim, é importante destacar que alguns fármacos, como glicocorticoides, progestágenos e anticonvulsivantes aumentam o risco de obesidade; os glicocorticoides ampliam o efeito de gliconeogênese, o catabolismo de proteínas e lipídeos, e os anticonvulsivantes, como o fenobarbital, realizam a inibição do centro de saciedade hipotalâmico, causando aumento da ingestão alimentar (MADDISON et al., 2010).

\section{Implicações metabólicas}

O tecido adiposo, atualmente, é considerado como um órgão endócrino ativo, nesse contexto, além de ser reservatório de energia, isolante térmico e protetor mecânico, também secreta uma gama de substâncias conhecidas como as adipocinas (BORGES, 2013), sendo que muitas delas são hormônios reguladores do metabolismo da glicose e lipídeos, como a leptina e a adiponectina, e outras são citocinas pró-inflamatórias, como as interleucinas (IL) e o fator de necrose tumoral $\alpha$ (TFN- $\alpha$ ) (ZORAN, 2010). Os pacientes obesos apresentam desequilíbrio da função endócrina do tecido adiposo, e isso pode trazer uma série de complicações locais e sistêmicas, que influenciam no desenvolvimento e agravamento dos problemas associados à obesidade (BORGES, 2013; CARVALHO, 2014).

A leptina é um hormônio responsável por reduzir a ingestão alimentar, sendo importante na saciedade, além de aumentar o gasto energético e regular o catabolismo dos carboidratos e lipídeos, sendo que em felinos está claramente ligada à sensibilidade à insulina (ZORAN, 2010). A concentração plasmática desse hormônio aumenta de acordo com o escore corporal, ou seja, quanto maior o grau de obesidade, maior o valor encontrado de leptina, devido a uma falha na sinalização desse hormônio a nível hipotalâmico, uma resistência ao hormônio, causada provavelmente por problemas no seu transportador ou na cascata de reações celulares (CARVALHO, 2014; FONSECA-ALANIZ et al., 2006).

A adiponectina está intimamente ligada ao metabolismo da glicose, aumentando a sensibilidade da insulina, além de possuir efeitos anti-inflamatórios, por suprimir a produção 
de TFN- $\alpha$ pelos macrófagos (ZORAN, 2010). Ao contrário da leptina, a adiponectina diminui sua concentração plasmática conforme aumenta a gordura corporal, devido à redução da expressão de seus receptores pelo feedback inibitório na sua secreção (MATTINSON; JENSEN, 2003; ZORAN, 2010).

Os adipócitos também secretam citocinas pró-inflamatórias e a obesidade é considerada como uma doença inflamatória crônica, devido a um aumento na produção dessas citocinas como TFN- $\alpha$ e IL-6 (ZORAN, 2010). Essas citocinas estão envolvidas na inflamação, na ativação do sistema imune frente a processos neoplásicos e infecciosos, atuam no desenvolvimento de resistência à insulina e, cabe ressaltar, também promovem o feedback inibitório na secreção de adiponectina (CARVALHO, 2014; ZORAN, 2010).

\section{Importância patológica da obesidade e consequências}

A obesidade é uma doença preocupante pois causa transtornos metabólicos, hormonais e inflamatórios (ZORAN, 2010). As transformações que a obesidade provoca no organismo do animal podem induzir o surgimento de diversas patologias (DEBASTIANI, 2018). A obesidade leva a uma redução na longevidade nos cães, um estudo realizado com Labradores Retrievers $(n=48)$ demonstrou que os cães que recebiam uma alimentação controlada pesaram menos e possuíam menores concentrações séricas de triglicerídeos; a insulina, a glicose e a vida média desses cães foi significativamente maior (13 anos) quando comparadas com as dos cães que não tinham restrição alimentar (11,2 anos). Qualquer sinal de doença crônica foi geralmente mais tardio para os cães com alimentação controlada (KEALY et al., 2002).

O excesso de peso é um desequilíbrio orgânico que põe em risco a saúde geral, por ser um fator altamente predisponente a muitas outras patologias como, por exemplo, a diabetes mellitus (MENDES et al., 2013). A obesidade altera as homeostases da insulina e da glicose; e os fatores relacionados à resistência insulínica em pacientes obesos são: a dislipidemia e as adipocinas, dentre elas, o TNF- $\alpha$, a IL-6, a leptina e a adiponectina (BORGES, 2013). Há de se considerar que as células beta do pâncreas continuam a produzir insulina, mas diminui a sensibilidade dos transportadores de glicose sensíveis à insulina (GLUT) causando hiperinsulinemia e diversos graus de intolerância à glicose (MENDES et al., 2013). Essa 
hiperinsulinemia persistente pode ser um fator importante no desenvolvimento ocasional de diabetes mellitus nos animais com obesidade (MENDES et al., 2013; SILVA et al., 2017).

A dislipidemia é uma complicação frequente associada à obesidade (CARVALHO, 2014). A hiperlipidemia pode ser definida como aumento de triglicerídeos, colesterol ou ambos no plasma (BORGES, 2013), os quais são os lipídeos séricos mais relevantes clinicamente (SILVA et al., 2017). Um estudo demonstrou que gatos com sobrepeso e obesidade apresentaram dislipidemias com aumento de colesterol lipoproteína de baixa densidade (LDL) e redução de colesterol lipoproteína de alta densidade (HDL) (MURANAKA et al., 2011).

A obesidade também pode afetar as funções cardíacas e pulmonares. Algumas doenças cardiovasculares, respiratórias e a hipertensão são influenciadas pela obesidade (BORGES, 2013; GERMAN, 2006). O aumento do peso corporal pode afetar o ritmo cardíaco, aumentando o volume do ventrículo esquerdo, a pressão sanguínea e o volume do plasma (SILVA et al., 2017). A insulina, a aldosterona e a norepinefrina aumentadas na obesidade, levam à retenção de sódio e consequente aumento do volume plasmático e pressão arterial, com isso, o excesso de volume pode causar aumento de pressão na parede do ventrículo esquerdo, levando à hipertrofia (BORGES; 2013; GERMAN, 2006; SILVA et al., 2017). Além disso, as adipocinas produzidas pelo tecido adiposo atuam na modulação de algumas alterações neuro-hormonais que conduzem ao aumento da pressão arterial na obesidade (SILVA et al., 2017). A obesidade em cães pode ser causa primária de desordens respiratórias, ou ser fator de risco adicional a problemas respiratórios pré-existentes, tais como: colapso de traqueia, bronquite crônica, síndrome braquicefálica e paralisia laríngea (GERMAN, 2006).

É evidente que a obesidade é um importante fator de risco para o desenvolvimento de doenças ortopédicas, sobretudo articulares, devido ao aumento de carga sobre as articulações (DEBASTIANI, 2018). Além disso, certas adipocinas pró-inflamatórias podem influenciar nas doenças articulares pela produção de metaloproteínas, enzimas degradadoras da matriz extracelular, como o colágeno e as glucosaminoglicanas, que degradam a cartilagem articular e diminuem a produção do líquido sinovial (BORGES, 2013). Dessa maneira, afirma-se que essas lesões articulares conduzem a uma redução de 
mobilidade e de gasto energético, o que pode afetar negativamente no tratamento (SILVA et al, 2017).

A obesidade também favorece o aparecimento de neoplasias, como tumores de bexiga, pele e mama em cadelas adultas (CARVALHO, 2014; GUIMARÃES; TUDURY, 2006). A fisiopatologia do câncer pode estar associada a algumas adipocinas, como a leptina, e mediadores da inflamação que estimulam o crescimento e desenvolvimento da lesão tumoral, por uma estimulação de IGF-1 (fator de crescimento semelhante à insulina-1), afetando assim os processos de divisão e diferenciação celular e angiogênese (BORGES, 2013; CARVALHO, 2014). De acordo com o estudo de German (2006), as neoplasias relacionadas ao excesso de peso em cães foram o carcinoma mamário e o carcinoma de células transicionais da bexiga.

\section{Diagnóstico}

Em cães e gatos, condições mais extremas de sobrepeso são facilmente reconhecidas. Entretanto, muitos métodos são pouco sensíveis a alterações mais sutis e tal fato dificulta uma intervenção precoce e o acompanhamento em programas de perda de peso (CARVALHO, 2014). O diagnóstico da obesidade geralmente envolve a associação entre a avaliação clínica do peso e a condição corporal (LAFLAMME, 2006). Existem métodos de diagnóstico por imagem que permitem a avaliação da composição corporal e a quantidade de gordura no animal, o que possibilita o conhecimento do grau de sobrepeso (CARVALHO, 2014).

A simples avaliação do peso é uma medida comumente utilizada na clínica veterinária, entretanto, esse método não explica a composição corporal, isto é, poderia ser o mesmo para indivíduos com sobrepeso e indivíduos com alto desenvolvimento muscular. Como caracterizou Robertson (2002), para cães, quando o peso está 10-25\% acima do peso ideal, é considerado com obesidade, mas estabelecer o peso ideal é uma tarefa problemática para o Médico Veterinário, pois os cães possuem muita variabilidade em níveis de peso e tamanho corporal. Para isso, em casos de raças puras, se tem a possibilidade de comparar com o peso padrão para a raça e estabelecer o peso ideal, ou pode-se comparar com um outro animal sem excesso de peso, com a mesma constituição e tamanho (GUIMARÃES; TUDURY, 2006; PÖPPL, 2018). Para felinos o peso pode variar de acordo com o porte, mas a faixa de 
normalidade para animais pequenos e médios corresponde de três a cinco quilos (MENDES et al., 2013).

O método mais utilizado, na rotina clínica, para a identificação da obesidade é a inspeção e palpação do animal, pelo método de escore de condição corporal (ECC), descrito por Laflamme (1997). Esse método é baseado na inspeção e palpação de locais com acúmulo de gordura corporal, principalmente nas regiões de proeminência óssea, como o gradil costal e base da cauda, além da visualização da silhueta e da cobertura de gorduras (LAFLAMME, 1997). De modo geral, o cão está com o escore ideal, quando possui as costelas facilmente palpáveis e apresenta a forma de ampulheta quando visto de cima (LAFLAMME, 1997). Abdômen abaulado a partir da última costela, com depósitos de gordura evidentes na inserção da cauda e cujo gradil costal não seja facilmente palpável, são indicativos de excesso de peso (LAFLAMME, 1997; RODRIGUES, 2011). Todavia, esse é um método subjetivo, que não capta alterações sutis de perda de peso, e que pode variar conforme avaliações de diferentes pessoas (CARVALHO, 2014).

Outro método disponível é a determinação do índice de massa corporal (IMC), um método muito utilizado em seres humanos para quantificar a massa corporal de pessoas adultas. Müller et al. (2008) fizeram a adaptação do índice de massa corporal humano para cães, denominado Índice de Massa Corporal Canino (IMCC), segundo a fórmula:

$$
\mathrm{IMCC}=\frac{\text { peso corporal }(\mathrm{kg})}{(\text { estatura } \mathrm{em} \mathrm{m})^{2}}
$$

De acordo com Müller et al. (2008), para mensurar a estatura do cão, utiliza-se uma fita métrica flexível, posicionando-a na base da nuca (articulação atlanto-occipital) e todo o dorso do animal até o solo, imediatamente atrás dos membros posteriores, passando e apoiando a fita sobre a base da cauda (última vértebra sacral), ficando a fita exatamente medial às tuberosidades ilíacas, sobre o dorso do animal; os resultados obtidos, para animais acima do peso foi de um IMCC entre 15,1 e 18,6 e para animais obesos acima de 18,7. Para felinos, Zoran (2009) estipulou o índice de massa corporal de felinos (IMCF), que permite 
saber a porcentagem de gordura utilizando uma fita métrica. A circunferência torácica ou perímetro torácico, será a circunferência do tórax, em centímetros, medida na altura da nona costela; a extensão do membro posterior será a distância, em centímetros, entre a patela esquerda e a tuberosidade calcânea do mesmo lado; com esses dados usa-se a fórmula:

$$
\text { IMCF }=\frac{1,5 \text { (circunferência torácica }- \text { membro posterior) }}{9}
$$

Já a morfometria é a avaliação das medidas corporais em vários sítios e baseia-se no princípio de que as proporções básicas do corpo estão relacionadas ao total de tecido magro, e que qualquer aumento de medida pode ser explicado pela adição de gordura (SILVA et al., 2017). Segundo o estudo de Guimarães (2009), as medidas morfométricas utilizadas são: altura da cernelha (AC), comprimento corporal (CC), membro pélvico direito $(\mathrm{MP})$, perímetro abdominal (PA), perímetro torácico $(\mathrm{PT})$ e perímetro da coxa $(\mathrm{PC})$. $\mathrm{E}, \mathrm{de}$ acordo com Rodrigues (2011) e Mawby et al. (2004), com as medidas morfométricas pode-se estabelecer a porcentagem de gordura corporal (\%GC) por meio das seguintes equações:

$$
\begin{aligned}
& \text { \%GC nos machos }=-1,4(\mathrm{MP} \text { em } \mathrm{cm})+0,77(\mathrm{PA} \text { em cm })+4 \\
& \% G C \text { nas fêmeas }=-1,7(\mathrm{MP} \text { em } \mathrm{cm})+0,93(\mathrm{PA} \text { em cm })+5
\end{aligned}
$$

Torna-se evidente que existe uma diversidade de métodos de diagnóstico para obesidade, entretanto, alguns não retratam a realidade da rotina clínica, como o DEXA, que é um método não invasivo que pode ser utilizado para estimar o conteúdo de gordura corporal. É realizado com raios $X$ em dois níveis de energia diferentes [70 e 140 quilovolts (kV)], para diferenciar o tipo e quantidade de cada tecido, assim com a atenuação exponencial dos diferentes níveis de energia dos raios $X$ emitidos, esse método consegue diferenciar os tecidos do corpo em osso, tecido de massa magra e macio (MAWBY et al., 2004). Apesar de 
ser o "padrão ouro," é um método que requer equipamentos específicos, por isso é mais utilizado em pesquisas, assim como a bioimpedância e ultrassonografia (RODRIGUES, 2011). Da mesma forma, outra possibilidade, é a técnica utilizando $\mathrm{D}_{2} \mathrm{O}$ (óxido de deutério), a qual baseia-se na diluição do $D_{2} O$ nos tecidos, que se permuta facilmente com a água, nesse sentido, ele se torna um marcador da condição corporal, já que a água corporal está predominantemente associada ao tecido magro, logo, esse método se torna uma medida indireta da massa corporal livre de gordura através da dosagem de óxido de deutério (MAWBY et al., 2004).

O escore de condição corporal ainda é o mais utilizado na rotina clínica, a praticidade e a confiabilidade do ECC foi demonstrada por Mawby et al. (2004) ao compararem quatro diferentes métodos de avaliação corporal: os métodos clínicos, escore de condição corporal e medidas morfométricas; e os métodos laboratoriais, DEXA (absorciometria de raios $\mathrm{X}$ de dupla energia) e $\mathrm{D}_{2} \mathrm{O}$ (óxido de deutério). Os autores encontraram uma boa correlação $\left(\mathrm{r}^{2}=\right.$ 0,92) entre o escore de condição corporal e o DEXA, assim como as medidas morfométricas com o DEXA.

\section{Tratamento}

O tratamento consiste no estabelecimento de metas para redução de peso e persistência, visando o peso ideal, pois demanda tempo e paciência por parte do tutor e do clínico veterinário (MENDES et al., 2013). Uma das maiores problemáticas no tratamento da obesidade é a falta de colaboração dos tutores (OLIVEIRA et al., 2010), já que o tratamento da obesidade passa por uma mudança no estilo de vida do animal e, por conseguinte, do tutor (CARVALHO, 2014). A conscientização e a comunicação com o tutor são de extrema importância para aumentar a adesão deste ao tratamento (PÖPPL, 2018). A terapia dietética constitui como o principal fator do controle de peso em cães e gatos, mas o aumento do exercício e o manejo comportamental são complementos essenciais (GERMAN, 2006).

Deve-se então, primeiramente, estabelecer um peso meta inicial, que não deve ser inferior a 15\% do atual, para evitar a ocorrência de transtornos metabólicos (MENDES et al., 2013; PÖPPL, 2018). A partir disso, pode-se estabelecer como meta a redução de 1 a $2 \%$ em cães e 0,5 a $1 \%$ em gatos, do peso total, por semana (LAZZAROTTO, 1999; PÖPPL, 2018). Em 
seguida, quando o paciente atingir o peso meta inicial, será determinada uma nova meta, sendo o segundo peso meta, e assim, sucessivamente, até o paciente atingir o peso meta final.

O princípio da terapia dietética é o fornecimento de dietas com total de energia menor do que o requerido para manter o peso corporal, propiciando um balanço energético negativo, visando induzir a mobilização de calorias do tecido adiposo (LAZZAROTTO, 1999; SILVA et al., 2017). As dietas para perda de peso são à base da restrição calórica de 60 e $70 \%$ das exigências energéticas para mantença dos cães e gatos, respectivamente (LAZZAROTTO, 1999). A necessidade diária total de calorias, para mantença, está em torno de $80 \mathrm{kcal} / \mathrm{kg}$ de peso corporal para os cães e $70 \mathrm{kcal} / \mathrm{kg}$ de peso corporal para os gatos. Com isso, após determinar o peso meta inicial, deve-se calcular as necessidades energéticas diárias a serem ingeridas por dia, em quilocaloria (kcal) (PÖPPL, 2018):

Cães e gatos $-(\mathrm{kcal} / \mathrm{dia})=70 \times(\text { Peso meta em kg })^{0,75}$

Estabelecido o valor da necessidade energética diária, é preciso escolher o tipo de alimentação. Existem no mercado dietas comerciais formuladas para a perda de peso que atendem as necessidades nutricionais do paciente, porém são menos calóricas quando comparadas com as rações para animais não obesos. Essas formulações hipocalóricas apresentam alta quantidade de fibras e proteínas, e baixa quantidade de carboidratos e gordura (JERICÓ et al., 2018). Isso porque o alimento deve conter elevado teor de proteínas, para prevenir a perda de massa magra durante o emagrecimento, assim como auxiliar no estímulo à saciedade, além de elevado teor de fibras, essencial para reduzir a densidade calórica do alimento (PÖPPL, 2018). A vantagem de se seguir uma dieta redutora de peso consiste no fato de que é possível balancear o volume e a caloria ingerida com menor risco de desenvolver transtornos nutricionais (JERICÓ et al., 2018). Alimentação natural também é uma alternativa bastante efetiva e interessante, visto que são utilizados ingredientes do dia a dia e que podem ser facilmente encontrados, com exceção de suplementos minerais, que devem ser empregados para suplementar as deficiências dos alimentos caseiros, por isso a 
grande questão quanto às dietas naturais é que devem ser balanceadas (CARFIOCI, 2013). Nas Tabelas 1 e 2 estão demonstradas duas formulações caseiras para programas de redução de peso para cães e gatos obesos sugeridas por Carfioci (2013).

Tabela 1 - Formulação de dieta caseira para cães obesos.

\begin{tabular}{|l|l|l|l|}
\hline Composição (\% da Matéria Seca) & Fórmula (\% da Matéria Original) & 40 \\
\hline Proteína Bruta & 30,75 & Arroz cozido & 15 \\
\hline Carboidrato & 53,95 & Músculo magro bovino ou peito de frango & 5 \\
\hline Extrato Etéreo & 6,41 & Fígado bovino & 15 \\
\hline Fibra Bruta & 4,74 & Cenoura & 23 \\
\hline Matéria Mineral & 4,09 & Vagem & 0,2 \\
\hline Umidade & 63,34 & Carbonato de cálcio & 0,6 \\
\hline Cálcio & 0,81 & Levedura de Cerveja & 1 \\
\hline Fósforo & 0,59 & Suplemento Mineral e Vitamínico & 0,1 \\
\hline & & Sal & 0,5 \\
\hline & & Óleo de Soja & $1,45 \mathrm{kcal} / \mathrm{g}$ \\
\hline
\end{tabular}

Tabela 2 - Formulação de dieta caseira para gatos obesos.

\begin{tabular}{|l|l|l|l|}
\hline \multicolumn{2}{|l|}{ Composição (\% da Matéria Seca) } & Fórmula (\% da Matéria Original) \\
\hline Proteína Bruta & 46,14 & Arroz cozido & 30 \\
\hline Carboidrato & 39,11 & Peito de frango & 30 \\
\hline Extrato Etéreo & 6,85 & Fígado bovino & 10 \\
\hline Fibra Bruta & 3,16 & Cenoura & 14 \\
\hline Matéria Mineral & 3,48 & Vagem & 14 \\
\hline Umidade & 62,14 & Carbonato de cálcio & 0,2 \\
\hline Cálcio & 0,72 & Levedura de Cerveja & 1 \\
\hline Fósforo & 0,70 & Suplemento Mineral e Vitamínico & 1 \\
\hline & & Sal & 0,2 \\
\hline & & Óleo de Soja & 0,5 \\
\hline & & Energia Metabolizável & $1,51 \mathrm{kcal} / \mathrm{g}$ \\
\hline
\end{tabular}

É necessário também calcular a quantidade de alimento a ser prescrita, uma regra de três simples define a quantidade a ser servida, por dia, em gramas (CARFIOCI, 2013). Logo, para o cálculo da quantidade de alimento, deve-se considerar a necessidade energética (NE) do animal, calculada anteriormente, e a energia metabolizável (EM), por grama, da dieta:

Quantidade de alimento $=$ NE (kcal por dia) $/$ EM por grama de dieta Quantidade de alimento $=\mathrm{x}$ gramas por dia 
Após calcular a quantidade a ser administrada em gramas por dia da dieta, deve-se calcular a quantidade de cada ingrediente da mistura, como no exemplo a seguir, para cães:

Ex: Arroz: Do total calculado (x gramas da dieta), 40\% será compreendido por arroz:

$$
\begin{aligned}
& \text { X gramas da dieta --------------100 \% (total) } \\
& \text { y gramas de arroz ------------ } 60 \% \text { (\% de arroz) } \\
& \qquad \text { y }=160 \text { gramas de arroz por dia }
\end{aligned}
$$

O ideal é fracionar a quantidade calculada em 3 a 5 refeições diárias, pois favorece maior gasto energético, e é fundamental que o alimento seja pesado em balanças de precisão para fornecimento da quantidade correta (PÖPPL, 2018). Ainda, segundo Guimarães e Tudury (2006) e Silva et al. (2017), o uso da L-carnitina ajuda a transportar ácidos graxos para o interior das mitocôndrias para conversão em energia, o que resulta na desejada perda de peso, assim como a suplementação com vitamina $A$, que tem provado ser efetiva, para normalizar a dosagem de leptina no soro, resultando em uma adequada perda de peso nos animais obesos. E a suplementação com ômega-3 como o EPA (ácido eicosapentanoico) e o DHA (ácido docosahexaenóico) é interessante para reduzir a produção de mediadores inflamatórios, característicos na obesidade (CARTOLANO, 2014).

O exercício físico, quando usado em combinação com terapia dietética, promove perda de gordura e pode ajudar na preservação do tecido muscular durante a terapia de perda de peso. Para a formulação de um programa de exercícios, devem ser considerados possíveis problemas de saúde como osteoartrites, e nos animais muito obesos é importante não os sujeitar a um aumento exagerado nos níveis de atividade, para evitar sobrecarregar articulações devido ao excesso de peso, portanto, passeios são uma ótima atividade física (GUIMARÃES; TUDURY, 2006). Inicialmente, o cão pode ser levado a dois ou três passeios curtos por dia; a duração de cada passeio pode ser gradualmente aumentada à medida que o cão se torne acostumado ao exercício e que a perda de peso comece a ocorrer (PÖPPL, 2018). 
Para felinos, é importante estimular a atividade para o tratamento da obesidade, porém não é uma tarefa fácil, já que isso envolve mudar a rotina do gato. O estímulo pode ser por meio de brinquedos como bolas, penas na ponta de hastes, lasers, túneis, prateleiras de acesso, estimular o animal a andar, pular e realizar atividades em geral é necessário, mas tudo deve ser realizado com calma (MENDES et al., 2013). O tutor deve ser insistente, pois gatos tendem a ser teimosos quanto a mudanças no estilo de vida, sendo importante ressaltar que todas essas atividades não devem ser um estresse excessivo ao animal (SILVA et al., 2017). A atividade física, além de estimular maior gasto calórico, possui outros benefícios para os pacientes, como reduzir a resistência insulínica secundária à obesidade, aumento da massa magra e redução da ansiedade (JERICÓ et al., 2018). Além desses efeitos, reduz a glicemia e o risco de diabetes mellitus, auxilia no controle da pressão arterial e estimula a lipólise por meio do estímulo à secreção de catecolaminas (PÖPPL, 2018).

É necessário, também, diagnosticar adequadamente as comorbidades e instituir a terapêutica indicada, além do acompanhamento do programa de emagrecimento por parte do Médico Veterinário, preferencialmente a cada 15 ou 30 dias, para o sucesso no tratamento.

\section{CONCLUSÃO}

A prevenção é a melhor maneira de combater a obesidade, uma vida saudável está intimamente relacionada à nutrição, por isso é essencial uma alimentação adequada, que atenda as exigências nutricionais do organismo, o hábito de exercícios físicos como caminhadas, corridas e brincadeiras que estimulem ainda mais o gasto energético. É muito importante conhecer a fisiopatologia da obesidade, conhecer suas complicações para saber identificar e indicar o melhor tratamento. 


\section{OBESITY: UNDERSTANDING THIS ORGANIC IMBALANCE IN DOGS AND CATS}

\section{ABSTRACT}

$\mathrm{O}$ besity is a pathological condition characterized by excessive accumulation of fat. It is one of the main pathologies observed in animals, being of increasing prevalence and influenced by several environmental and genetic factors, such as diet, physical activity, age, gonadal status, breed and endocrinopathies. It is a condition that favors the animal to various complications in health, such as reduced longevity, problems in joints, neoplasias and diabetes mellitus. There are several methods that help in the diagnosis of this pathology and are important for treatment. Thus, this review aims to expose and discuss the possible etiologies and complications, the methods used for the diagnosis of obesity and treatment.

Keywords: Overweight. Nutritional disturbance. Canids. Felids.

\section{OBESIDAD: COMPRENDIENDO ESE DESEQUILIBRIO ORGÁNICO EN PERROS Y GATOS}

\section{RESUMEN}

a obesidad es una condición patológica caracterizada por el acúmulo excesivo de grasa, siendo una de las principales patologías observadas en perros y gatos. Es una enfermedad de prevalencia creciente y está influenciada por diversos factores, ambientales y genéticos, como dieta, actividad física, edad, status gonadal, raza y endocrinopatías. Es una condición que favorece al animal a diversas complicaciones en la salud, como reducción de la longevidad, problemas en articulaciones, neoplasias y diabetes mellitus. Existen diversos métodos que auxilian en el diagnóstico de esta patología y que son importantes para el tratamiento. Ante todo, ésta revisión tiene por objetivo exponer y discutir las posibles etiologías y complicaciones, así como los métodos utilizados para el diagnóstico de la obesidad y el tratamiento.

Palabras clave: Sobrepeso. Trastorno nutricional. Canidos. Félidos. 


\section{REFERÊNCIAS}

APTEKMANN, K. P.; SUHETT, W. G.; SOUZA, G. B.; et al. Aspectos nutricionais e ambientais da obesidade canina. Ciência Rural, v. 44, n. 11, p. 2039-2044, 2014.

BLAND, I. M.; GUTHRIE-JONES, A.; TAYLOR, R. D.; et al. Dog obesity: veterinary practices' and owners' opinions on cause and management. Preventive Veterinary Medicine, v. 94, n. 3-4, p. 310-315, 2010.

BORGES, L. N. P. M. Fatores relacionados à obesidade em cães: uma revisão introdutória. Brasilia: UnB, 2013. 35p. Monografia (Trabalho de Conclusão de Curso), Curso de Medicina Veterinária, Faculdade de Agronomia e Medicina Veterinária, Universidade de Brasília, 2013. Disponível em:

<http://www.bdm.unb.br/bitstream/10483/5937/1/2013_LuizaNobrePinheiroMontandonB orges.pdf $>$.

BRUNETTO, M. A.; NOGUEIRA, S.; SÁ, F. C.; et al. Correspondência entre obesidade e hiperlipidemia em cães. Ciência Rural, v. 41, n. 2, p. 266-271, 2011.

CARFIOCI A. C. Dietas Caseiras para Cães e Gatos: Fórmulas Práticas e Princípios de Utilização. São Paulo: UNESP, 2013. 17p. Apostila (Serviço de Nutrição Clínica), Departamento de Clínica e Cirurgia Veterinária, Faculdade de Ciência Agrárias e Veterinárias, Universidade Estadual Paulista, 2013. Disponível em:

<https://www.fcav.unesp.br/Home/departamentos/clinicacv/AULUSCAVALIERICARCIOFI/Die tas-caseiras.pdf $>$.

CARTOLANO, F. C. Efeito do ácido graxo ômega 3 sobre a composição corporal, controle glicêmico e risco cardiovascular de indivíduos adultos e idosos. São Paulo: USP, 2014. Dissertação (Mestrado em Saúde Pública), Faculdade de Saúde Pública, Universidade de São Paulo, 2014.

CARVALHO, G. L. C. Obesidade em cães e gatos: complicações metabólicas. Porto Alegre: UFRGS, 2014. 6p. Seminário (Disciplina Transtornos Metabólicos dos Animais Domésticos do Programa de Pós-Graduação em Ciências Veterinárias), Universidade Federal do Rio Grande do Sul, 2014.

DEBASTIANI, C. Epidemiologia da obesidade canina: fatores de risco e complicações. São Paulo: UNESP, 2018. 82p. Dissertação (Mestrado em Medicina Veterinária), Programa de Pós-Graduação em Medicina Veterinária, Faculdade de Medicina Veterinária e Zootecnia, Universidade Estadual Paulista, 2018. Disponível em:

<https://repositorio.unesp.br/bitstream/handle/11449/154442/debastiani_c_me_bot.pdf?s equence $=3$ \&isAllowed $=y>$.

FEITOSA, F. L. F. Semiologia veterinária: A arte do diagnóstico. São Paulo: Roca, 2014. 752p. 
FONSECA-ALANIZ, M. H.; TAKADA, J.; ALONSO-VALE, M. I. C.; et al. O tecido adiposo como centro regulador do metabolismo. Arquivos Brasileiros de Endocrinologia \& Metabologia, v. 50, n. 2, p. 216-229, 2006.

GERMAN, A. J. The growing problem of obesity in dogs and cats. Journal of Nutrition, v. 136, n. 7, p. 1940-1946, 2006.

GUIMARÃES, A. L. N.; TUDURY, E. A. Etiologias, conseqüências e tratamentos de obesidades em cães e gatos-revisão. Veterinária Notícias, v. 12, n. 1, p. 29-41, 2006.

GUIMARÃES, P. L. S. N. Conformação corporal e bioquímica sanguínea de cadelas adultas castradas alimentadas ad libitum. Goiânia: UFG, 2009. 142p. Tese (Doutorado em Ciência Animal), Escola de Veterinária, Universidade Federal de Goiás, 2009.

JEUSETTE, I.; GRECO, D.; AQUINO, F.; et al. Effect of breed on body composition and comparison between various methods to estimate body composition in dogs. Research in Veterinary Science, v. 88, n. 2, p. 227-232, 2010.

JERICÓ, M. M.; LORENZINI, F.; KANAYAMA, K.; et al. Obesidade Canina e felina. Associação Brasileira de Endocrinologia Veterinária, 2018. Disponível em: <https://vetsmartparsefiles.s3.amazonaws.com/63ed1193d11ff778e46c342651cada12_streaming_attachmen t.pdf> .

JERICÓ, M. M.; SCHEFFER, K. C. Aspectos epidemiológicos dos cães obesos na cidade de São Paulo. Revista Clínica Veterinária, v. 7, n. 37, p. 25-29, 2002.

KEALY, R. D.; LAWLER, D. F.; BALLAM, J. M.; et al. Effects of diet restriction on life span and age-related changes in dogs. Journal of the American Veterinary Medical Association, v. 220, n. 9, p. 1315-1320, 2002.

LAFLAMME, D. P. Development and validation of a body condition score system for dogs: a clinical tool. Canine Practice, v. 22, n. 4, p. 10-15, 1997.

LAFLAMME, D. P. Understanding and managing obesity in dogs and cats. Veterinary Clinics of North America: Small Animal Practice, v. 36, n. 6, p. 1283-1295, 2006.

LAZZAROTTO, J. J. Relação entre aspectos nutricionais e obesidade em pequenos animais. Revista da Universidade de Alfenas, v. 5, p. 33-35, 1999.

LUND, E. M.; ARMSTRONG, P. J.; KIRK, C. A.; et al. Prevalence and risk factors for obesity in adult cats from private US veterinary practices. The International Journal of Applied Research in Veterinary Medicine, v. 3, n. 2, p. 88-96, 2005. 
LUND, E. M.; ARMSTRONG, P. J.; KIRK, C. A.; et al. Prevalence and risk factors for obesity in adult dogs from private US veterinary practices. The International Journal of Applied Research in Veterinary Medicine, v. 4, n. 2, p. 177-186, 2006.

MADDISON, J.; PAGE, S.; CHURCH, D. Farmacologia Clínica de Pequenos Animais. Rio de Janeiro: Elsevier, 2010.

MALTA, D. C; ANDRADE, S. C.; CLARO, R. M.; et al. Evolução anual da prevalência de excesso de peso e obesidade em adultos nas capitais dos 26 estados brasileiros e no Distrito Federal entre 2006 e 2012. Revista Brasileira de Epidemiologia, v. 17, supl. 1, p. 267-276, 2014.

MATTINSON, R.; JENSEN, M. The adipocyte as an endocrine cell. Current Opinion in Endocrinology, Diabetes and Obesity, v. 10, n. 5, p. 317-321, 2003.

MAWBY, D. I.; BARTGES, J. W.; D'AVIGNON, A. Comparison of various methods for estimating body fat in dogs. Journal of the American Hospital Association, v. 40, n. 2, p. 109114, 2004.

MENDES, F. F.; RODRIGUES, D. F.; PRADO, Y. C. L.; et al. Obesidade Felina. Enciclopédia Biosfera, v. 9, n. 16, p. 1602-1625, 2013.

MURANAKA, S.; MORI, N.; HATANO, Y.; et al. Obesity induced changes to plasma adiponectin concentration and cholesterol lipoprotein composition profile in cats. Research in Veterinary Science, v. 91, n. 3, p. 358-361, 2011.

MÜLLER, D. C. M.; SCHOSSLER, J. E.; PINHEIRO, M. Adaptação do índice de massa corporal humano para cães. Ciência Rural, v. 38, n. 4, p. 1038-1043, 2008.

MAO, J.; XIA, Z.; CHEN, J.; et al. Prevalence and risk factors for canine obesity surveyed in veterinary practices in Beijing, China. Preventive Veterinary Medicine, v. 112, n. 3-4, p. 438442, 2013.

NELSON, R. W.; ELLIOTT, D. A. Distúrbios Metabólicos e Eletrolíticos. In: NELSON, R. W.; COUTO, C. G. Medicina Interna de Pequenos Animais. Rio de Janeiro: Elsevier, 2006. Cap. 54, p. 781-792.

OLIVEIRA, M. C.; NASCIMENTO, B. C. L.; AMARAL, R. W. C. Obesidade em cães e seus efeitos em biomarcadores sanguíneos-revisão de literatura. PUBVET, v. 4, n. 13, p. 795-801, 2010.

PÖPPL, A. G. Obesidade em cães e gatos. PromeVet, ciclo 3, v. 4, 2018. 67p. Disponível em: $<w w w$. researchgate.net/publication/326211850>.

ROBERTSON, I. D. The association of exercise, diet and other factors with owner-perceived obesity in privately owned dogs from metropolitan Perth, Western Australia. Preventive Veterinary Medicine, v. 58, n. 1-2, p. 75-83, 2002. 
RODRIGUES, L. F. Métodos de avaliação da condição corporal em cães. Goiânia: UFG, 2011. 28p. Seminários Aplicados (Disciplina do Programa de Pós-Graduação em Ciência Animal), Escola de Veterinária e Zootecnia, Universidade Federal de Goiás, 2011. Disponível em: <https://files.cercomp.ufg.br/weby/up/67/o/semi2011_Leticia_Furtado_2c.pdf> .

SILVA, S. F.; BRITO, A. K. F.; FREIRE, B. A. A.; et al. Obesidade canina: revisão. PUBVET, v. 11, n. 4, p. 371-380, 2017.

WHO - WORLD HEALTH ORGANIZATION. Global Health Observatory (GHO) data. Overweight and obesity. Adults aged 18+, 2016. Disponível em:

<https://www.who.int/gho/ncd/risk_factors/overweight_text/en/> .

ZORAN, D. L. Feline obesity, clinical recognition and management. Compendium: Continuing Education for Veterinarians, p. 284-291, 2009. Disponível em: <https://vetfoliovetstreet.s3.amazonaws.com/mmah/9f/5b944b43a24ab1a4e6243101957d6f/filePV_31_06_ 284.pdf>.

ZORAN, D. L. Obesity in Dogs and Cats: A Metabolic and Endocrine Disorder. Veterinary

Clinics of North America: Small Animal Practice, v. 40, n. 2, p. 221-239, 2010.

Autor para correspondência: Antônio Gonçalves de Andrade Júnior. Universidade Federal de Pelotas, Faculdade de Veterinária, Campus Universitário, Capão do Leão (RS), Brasil. antonio_3@icloud.com 